\title{
Can nurse practitioners offer a quality service? An evaluation of a year's work of a nurse led minor injury unit
}

\author{
Abbas F Mabrook, Barbara Dale
}

\begin{abstract}
Objective-To evaluate activities of a nurse led minor injuries unit based in a community hospital situated eight miles away from its sister district hospital in Crawley to demonstrate whether nurses can provide an alternative service.

Design-"Walk in" service provided by emergency nurse practitioners (ENPs) working within protocols and parameters with supervision from the accident and emergency consultant during the period of May 1995 to April 1996.

Results-Within the 12 month period, ENPs treated 6944 new patients. Altogether 234 patients were transferred to Crawley Hospital for further treatment. The ENPs reviewed 1611 patients and the consultant 1342; 1945 patients required radiographs and 793 were given medication; 553 patients were referred to other health care professionals. Almost all patients were happy to see a nurse and all were satisfied with the treatment they received.

Conclusion-With careful planning, adequate supervision, and support from multidisciplinary teams nurses can provide a worthwhile and effective service for a local community.

( 7 Accid Emerg Med 1998;15:266-268)
\end{abstract}

Keywords: nurse practitioners; minor injury unit; quality

Concerns about the "casualty unit" at Horsham Community Hospital working in isolation without senior medical supervision made it necessary to review the service being provided. A survey of the facilities, attendances, and activities of the unit was conducted. This showed that $80 \%$ of injuries treated were minor trauma. The survey also revealed a need to retain a service at Horsham as the large numbers of attendances would create an extra workload for the accident and emergency (A\&E) department at Crawley Hospital situated eight miles away. Several options were considered as to how best to maintain the service including the possibility of a nurse unit as minor injuries could be assessed and treated by nurses.

The concept of nurse practitioners is not a new one. ${ }^{1}$ For many years senior nurses in outlying community hospitals for all intents and purposes have carried out the role when using their clinical judgment to decide when and where to refer patients to medical personnel. In recent years this role has been utilised in various ways in $A \& E$ departments for the benefit of patients. ${ }^{23}$ Current developments in the provision of minor injury care showed that nurses could successfully provide such a service. ${ }^{4}$ In the light of this Crawley Horsham NHS Trust took the decision to overcome current difficulties and to continue providing a minor trauma service at Horsham by using emergency nurse practitioners (ENPs) supervised by the $A \& E$ consultant based at Crawley Hospital. The senior house officer on call for care of the elderly would be called upon in an emergency. This service started in May 1995.

The decision to use ENPs to deliver the service provoked considerable pressure from the local community who wanted the status quo maintained. Both lay persons and professionals questioned the ability of nurses to deliver an adequate service. The local ambulance service expressed concerns of possible increased demands to transfer patients to Crawley Hospital and the on site outpatient departments feared that an increased number of referrals to their clinics would create an overload to the already very busy clinics. It was therefore important to closely monitor the new service to ascertain whether nurses could provide an alternative worthwhile service for the community.

\section{Methods}

A public awareness campaign was conducted before the start of the service. All local general practitioners were informed of the change in service. Leaflets were distributed to households in the local area stating the types and extent of injuries the ENPs could treat. Posters were distributed to schools, clinics, and to general practitioner surgeries. The local papers and radio station also played their part in educating the local community as to the type of service the ENPs would provide.

Considerable care and effort was taken to select suitable experienced nurses to run the unit. Nurses with a minimum of five years $A \& E$ experience were appointed and they had to be fully committed to the new venture. The nurses attended a formal three month ENP course. The A\&E consultant provided additional training at Crawley Hospital. This consisted of six weeks of observation plus six weeks of supervised work of assessment, diagnosis, and treatment of various minor injuries.

The role of the ENP was based on the Royal College of Nursing guidelines ${ }^{5}$ and the United 
Kingdom Central Council directive. ${ }^{6}$ The nurses carried out a full assessment, provided treatment, care, and discharge or referred patients to other health care professionals. Health promotion advice was incorporated in the overall care given.

Wide ranging protocols and parameters were written and agreed based on activities highlighted in the review. These included radiography, medication, treatment, and referral protocols. Within the previously agreed protocols, ENPs could request and interpret radiographs of upper limb (shoulder to fingers), lower limb (knee to toes), and chest and abdomen in cases of ingestion of foreign bodies, for example batteries. Patients under 2 years and pregnant women were excluded. Within the agreed treatment protocol ENPs were allowed to administer simple analgesia, antibiotics, and tetanus toxoid. Stronger analgesia required for patients with displaced fractures was prescribed by the senior house officer on call for care of the elderly.

During the first year all radiographs requested by the ENPs were retained in the unit with the patient' notes for review by the A\&E consultant. This formed part of the monitoring of the nurses' work and ongoing education. The films were then returned to the $x$ ray department for a formal report by a radiologist.

Patients who had their radiographs misinterpreted by the ENPs were informed by telephone and a consultant review appointment was given. All patients diagnosed as having possible fractures, that is false positives, were automatically given a consultant review appointment. In both instances of misinterpretations of radiographs, patients' symptoms were treated.

Seven part time staff provide a "walk in" minor injuries treatment service between the hours of 9 am and $5 \mathrm{pm}$ Monday to Friday excluding Bank Holidays. The staff comprises four ENPs (F grade), two support nurses (D grade), and a receptionist. Two ENPs and a support nurse cover each shift. The A\&E consultant based at Crawley Hospital provides medical advice, support, and supervision. The consultant holds twice weekly clinic sessions in the unit to review patients' notes and radiographs, all patients with simple fractures treated by the ENPs, and any other soft tissue injury referrals. ENPs may discuss cases and or obtain advice from senior medical personnel in Crawley $A \& E$ department at any time.

In order to reflect accurately the day to day activities of the unit, special forms were devised to facilitate concurrent audit. Data collection was carried out by the nurses on a daily basis.

\section{Results}

Patients of all ages were assessed. During the period May 1995 to April 1996 a total of 9897 patients were treated. Of these 6944 were new patients and 1611 were ENP follow up patients. The consultant reviewed 690 patients with fractures and 652 patients with soft tissue injuries. Altogether 3427 of the patients treated were under 17 years of age; $96 \%$ of patients were self referred. One hundred and one patients treated were referred by local general practitioners and 169 were referred by other professionals, for example physiotherapists, opticians, and other hospitals. All children under 1 year were referred to the paediatric department at Crawley Hospital. Other referrals from the unit included 352 patients to the physiotherapy department, 159 to their general practitioner, seven to their dentist, 68 to the eye clinic, and 25 to the ear, nose, and throat clinic. Of the 6944 new patients treated 234 were transferred to Crawley A\&E department for further management. These included head injuries, fractures with displacement, lacerations involving deep structures, Achilles tendon rupture, compartment syndrome, deep venous thrombosis, facial injuries, and chest pains. Transportation by ambulance was required for 28 of the referrals to Crawley $A \& E$ department. Of the 760 fractures treated, 70 were referred to Crawley A\&E department by the ENPs; 690 fractures were treated by ENPs and were followed up in the unit by the consultant during the twice weekly clinic sessions. Seventy four of the ENP follow up fractures were referred to the fracture clinic by the consultant.

Of the 8555 patients treated by ENPs 1945 required radiography investigations. There were 22 missed fractures and 57 false positive readings. The misinterpreted radiographs were 10 flake fractures of fingers, six flake fractures of toes, two flake fractures of ankle, two possible crack fracture of head of radius, one possible greenstick fracture of clavicle, and one possible crack fracture of epiphyseal plate of tibia.

A patient satisfaction questionnaire was conducted over a period of three weeks, six months after the start of the service. From 313 questionnaires handed out 269 were returned. Analysis of the returned questionnaires indicated that 263 patients examined by a nurse were satisfied with the treatment or advice given and had no objections to being treated by a nurse. Six patients stated that they would have preferred to be treated by a doctor, although they had no complaints about the treatment they received. There were no written complaints during the first year.

\section{Discussion}

Careful planning and preparation have been key factors to the apparent success of the nurse led unit. Using information based on previous service provision and existing nurse units, a service based on the needs of the local community and facilities available was established. This is reflected in the wide range of previously agreed protocols that determine the scope of practice and level of intervention. Allowing the ENPs to request radiographs and to administer certain medications within the agreed protocols were major factors in determining the apparent effectiveness of the service as these allowed the nurses to treat and discharge the majority of patients who attended without the need for referral to medical personnel. During its first year the unit has provided treatment for 9897 . The level and breath of activities show that the local com- 
munity has used the service, and interestingly clients of all ages attended for treatment. Altogether $98 \%$ of the patients surveyed were happy to be seen by a nurse and were satisfied with the treatment they received. The level of transfers, referrals to clinics, and the number of clients treated by the ENPs suggest that all the fears and scepticism expressed before the start of the service appear to be unfounded.

The authors conclude that this study has demonstrated that given suitable training, protocols, and close liaison with a neighbouring
A\&E department, nurses can provide an effective and worthwhile locally based service.

1 Stilwell B, Greenfield S, Drury V, et al. A nurse practitioner in general practise: working styles and patterns of consultations. Journal of Royal College of General Practitioners 1987;37:154-7.

2 Potter T. A real way forward in A\&E: developing the nurse practitioner role. Professional Nurse 1990;5:586-8.

3 Woolwich C. A wider frame of practice. Nursing Times 1992;88:34-6 (11 Nov)

4 Baker B. Model methods. Nursing Times 1993;89:33-5 (24 Nov).

5 Royal College of Nursing A\&E Association and Emergency Nurse Practitioner Special Interest Group. Emergency nurse practitioners: recommendations of special interest group. practitioners: recommenda

6 United Kingdom Central Council. The scope of professional practice. London: UKCC, 1992.

\section{Hong Kong Academy of Medicine First International Congress, Hong Kong 26-29 November 1998}

\section{Challenges to Specialists in the 21st Century}

Plenary lectures: Challenges to the specialist beyond 2000; Evidence based medicine; Survival of the generalist in a specialist world; The quest for transplantation tolerance; The training of our specialists into the next millennium; Pain-free state: is it possible?

The scientific programme includes symposia on trauma, pain management, and substance abuse.

For further information, contact the Congress Secretariat, Hong Kong Academy of Medicine, First International Congress, GPO Box 41, Hong Kong; tel: 8522515 5737; fax: 8522505 3149; e-mail: hkam@hkam.org.hk 